\title{
An Online Acquaintance Community: The Emergence of Chinese Virtual Civility
}

For citation:

Tian, Xiaoli and Yanan Guo. 2020 (accepted and forthcoming). "An Online Acquaintance

Community: The Emergence of Chinese Virtual Civility.”Symbolic Interaction.

Xiaoli Tian

The University of Hong Kong

Yanan Guo

The University of Hong Kong

\section{Author Note}

Xiaoli Tian ${ }^{1}$, Associate Professor, Department of Sociology, The University of Hong

Kong, Hong Kong. Email: xltian@ @ku.hk.

Yanan Guo, PhD Candidate, Department of Sociology, The University of Hong Kong,

Hong Kong. Email: angelguo@ connect.hku.hk.

\footnotetext{
Direct all correspondence to Xiaoli Tian, Department of Sociology, The University of Hong Kong, Room 922, The Jockey Club Tower, Centennial Campus, The University of Hong Kong, Pokfulam, Hong Kong; e-mail: xltian@hku.hk.
} 


\title{
An Online Acquaintance Community: The Emergence of Chinese Virtual Civility
}

\author{
ABSTRACT \\ How is social etiquette performed when offline interpersonal interactions are mediated \\ online? Sixty semi-structured interviews with WeChat users, China's most popular social \\ media platform, show that the app's technical designs facilitated a set of online etiquette rules \\ which reproduced those of acquaintance communities. Three dimensions of social etiquette \\ rules were considered and observed: respect, elegance, and tidiness. Users honored the face \\ of others and avoided causing others to lose face, often presented a positive but restrained \\ self-image, and strived to preserve the tidiness of the online public space by avoiding the \\ sharing of and exposing others to "negative energy".
}

Keywords: online interaction, social etiquette, technical affordances, social media, culture, China

\section{INTRODUCTION}

Social etiquette constitutes the social norms that govern individual behavior (such as body movement and language) in public space and proper ways of social interaction. It has also been a popular area of research in offline face-to-face $(\mathrm{FtF})$ interactions (e.g., Elias 1939/1978; Goffman 1955, 1967; Pan 2000; Yan 2017). Social etiquette rules are embedded in the social structure and culture of a society, and since the definitions and interpretations of 
good manners and politeness vary between societies, etiquette rules are characterized by their varied histories, cultures, and institutional structures. For example, many developed Western societies that are characterized by high levels of population flow and migration, such as the United States, premise social etiquette on a "stranger society" where everyone is independent and considered as equal individuals (Simmel 1922, 1950). Therefore, independence and selfreliance are guiding principles of good manners, and there is little variation between interacting with families and with strangers (Yan 2017; Zhao 2016).

China, however, has long been described as an "acquaintance society" (Fei 1948; Liu 2002, 2014; Wang 2006), where interpersonal relationships are highly local and there is limited mobility among group members. For example, people grow up with the same group of individuals, reside nearby, are on familiar terms with each other's social contacts, and overall, there is little choice in determining one's interactants (Zhai 2004). Thus, an acquaintance society prioritizes giving face to others, showing respect to seniors, and maintaining interpersonal harmony (Yan 2017; Zhao 2016).

Over the last forty years, China's traditional acquaintance society has gradually transformed into a quasi-acquaintance society due to the drastic changes in social structures along with urbanization and modernization. A quasi-acquaintance society features hybrid relationships that incorporate strangers and acquaintances (Liu 2014b; see also Chen 2019; Zhai 2004), and these hybrid relationships influence how people interact. At the same time, as communication is increasingly mediated online, social networking sites may also present new challenges for relationship management and interactional norms, such as the lack of 
physical co-presence between interactants, ease of documenting past conversations, and the $n$-adic nature of online disclosures, where the potential number of audience $(n)$ one is interacting with is uncertain, diverse, and invisible (Tian and Menchik 2016). These shifts have fundamentally influenced how we define and preserve our backstage lives and created new challenges and expectations for digital platforms and its users (Armond 2018).

Technology has the power to generate social restructuring, impact human lives, and make social change by what it wants, drives, and demands (Barley 1986; Kelly 2010; Neff et al. 2012). In this study, we examine the following questions: What norms or social etiquette do Chinese WeChat users follow when interacting in an online acquaintance community? How does technology facilitate and mediate users' perception and practice of such etiquette? What are the implications and consequences of their practice of those etiquette rules?

To answer these questions, we focus on WeChat, the most popular mobile phone application in China with over 1.16 billion monthly active users (Tencent 2020). Almost 95\% of WeChat contacts are offline acquaintances (CNNIC 2016) and 86.9\% of WeChat users (CNNIC 2018) who share posts with contacts and respond to others' posts regularly use one of its most popular functions called "WeChat Moments" (pengyou quan, literally translated as "friends" circle"). Because users cannot see interactions among or information posted by friends of friends, amongst other technical affordances in the app, Moments is a striking reproduction of the small networks that make up an acquaintance community and Moments replicates the patterns of everyday interactions among offline friend groups. 
Our 60 semi-structured interviews conducted with WeChat users reveal that, over time, users developed and observed a set of etiquette rules that guided self-presentation, treatment of others, and maintenance of this public space. As Davis (2020) argued, social media design can perpetuate or resist certain values and norms. We find that social etiquette in the online acquaintance community emphasized giving face to others, and with the facilitation of technology, those with different relationships were treated differently. Such practices have led to the emergence of "Chinese virtual civility", which has fostered a set of new norms in interpersonal interactions online.

\section{SOCIAL ETIQUETTE AND INTERPERSONAL INTERACTION IN THE CHINESE}

\section{CONTEXT}

Zhao (2016) differentiated etiquette into three different dimensions: respect, elegance, and tidiness. "Respect" is about showing amicability to others through polite language. "Elegance" is the expression of a good self-image, mainly through appearance and behavior. In interpersonal communication, appearance is prioritized because physical image usually signifies the degree of refinement and shows effort being made to present a positive image. "Tidiness" is to keep the surrounding environment clean, peaceful, and orderly when interacting with others. Since emotions are affected by surroundings, one's attitude towards a social interaction environment indirectly conveys one's attitude towards others and reflects one's refinement and civility. 
While these three elements are somewhat universal, their definitions and interpretations vary among societies. To describe Chinese interpersonal relationships, Fei Hsiao-Tong (1948/1992) coined the term “differential modes of association" (chaxu geju) and described the relationship system in Chinese society as "the ripples formed from a stone thrown into a lake, each circle spreading out from the center becomes more distant and [...] more insignificant" (1992:65). These circles are families, kinship, neighbors, acquaintances, and strangers who are given different weight based on the caliber of the relational ties. Fei's understanding of the Chinese society was echoed by that of Morton Fried (1953), who proposed that networks of kinship, friendships, neighbors, and villagers are the basic units for mobilizing resources and collective action in Chinese society.

These differential modes of association mean that the Chinese often adhered to relationship-based moral standards and behavioral codes (Fei 1948; Hsu 1953; Hwang 1987). Maintaining these relationships requires ongoing contact and reciprocal exchange, and such interactions face constant scrutiny and evaluation by others in the same network. Moreover, since future interactions are anticipated, individuals are obliged to maintain harmonious relationships and avoid conflict. Indeed, the importance of face and facework in China is a major difference in social etiquette when compared to the Western context. Despite the universal relevance of face (Brown and Levinson 1987; Goffman 1967), face has a more salient importance in Chinese interactions (Hsu 1996; Hwang 1987).

In Chinese society, face is tied to guanxi (relationship) and renqing (obligations among guanxi members) (Hwang 1987). To maintain interpersonal harmony, a requested favor from 
another network member may need to be accommodated to give face to them (Hwang 1987). Those who do not follow the principles of face and renqing, for example by failing to give face to others or turning down requests for favors when asked, will be accused of disrupting the harmony in relationships and violating the norms of renqing. Worse, those individuals could be viewed as rude and impolite; people who are incapable of basic civility ( bu hui zuo ren), or even immoral, and thus could be ostracized from mainstream society (Hsu 1996; Hwang 1987; Kinnison 2017). Historically, the social etiquette involved in facework is tied to China being an acquaintance society (Zhai 2004). Since relationships were expected to have longevity, cultivating harmonious interpersonal relationships was a priority. In fact, the survival of the community rested on mutual dependence and collaboration.

Notably, the above social etiquette rules, which are used as principles of social interaction, changed when China underwent societal development. A series of wars and revolutions from the 1840 s to the 1940 s ushered in modernization, which gradually transformed traditional society in China. The establishment of the People's Republic of China in 1949 economically and politically transformed the country. Yet, these transformations, such as the implementation of a planned economy and a strict household registration system (hukou system), largely preserved the features of an acquaintance society in both rural and urban China (Jiao 2015).

Economic reforms and its opening up since 1978 transformed China from an acquaintance society to one that started to embrace the characteristics of a stranger society or a semi-acquaintance society. The population flow from rural to urban areas has increased 
over the past forty years and urbanization has also been on the rise. An average of six to eight million new migrant workers look for jobs outside their hometown each year (Han 2006). China has been experiencing the largest and most rapid urbanization in history since 1949, with an average annual increase of $0.71 \%$. At the end of 2018 , the amount of urbanization had reached nearly $60 \% .^{2}$ Meanwhile, a large number of practitioners working for state enterprises (guoyou qiye) and administrative organs of the government (jiguan danwei) in urban China left their jobs and went into business (xia hai) for themselves (Liu 2014b). These changes, accompanied by advancements in transportation technologies, led to a large-scale population flow between rural and urban China, which permanently altered the original social units and interactive routines from the traditional acquaintance society (Jiao 2015; Wu 2011).

Additionally, in the face of a new market economy with much higher risks, values are increasingly influenced by market principles such as rationality, calculability, competition for interests, fairness, and openness in interactions, many of which diverge from the interactive norms of an acquaintance society (Jiao 2015). However, despite these observed changes, research work in social network analyses has provided evidence on the importance and perpetuation of kinship networks and other overlapping egocentric networks in everyday social and economic activities in Chinese society (see Bian 1997, 2019; Chang 2010; Kipnis 1997; Lin 2001; Michelson, 2007; Yan 1996; Yang 1994, as cited in Burt et al. 2019). For example, Bian (1994) pointed out that guanxi played an important role in job searching and social mobility in urban China in the post-reform era. Other research found that in China and

\footnotetext{
2 See http://www.gov.cn/xinwen/2019-08/16/content_5421576.htm.
} 
Taiwan, kinship ties influence socioeconomic institutions to a greater extent than in the United States (Lin, Fu, and Chen 2013), which applies to the networks of urbanites in China (Wang, Liao, and Xiao 2014). More recently, a comparison study conducted by Burt and Burzynska (2017) showed substantial evidence that guanxi was a core characteristic of networks around Chinese entrepreneurs and two-thirds of the study's participants' key contacts were guanxi ties. At the micro level, it can be argued that the interactional, relational, and psychological aspects of the acquaintance society still persist, and elements of that society have been preserved (Liu 2014a, 2014b; Ma 2015). Consequently, many factors regarding how people relate to each other in contemporary China have not fundamentally changed (Liu 2014a, 2014b).

This then leads to important questions about the development of interactional and relational norms of Chinese users in an online community, where interpersonal interactions are broadcasted to larger audiences than everyday FtF social exchanges, and presented with multiple invisible audiences from various social networks. It would thus be important to study how digital technologies and technical affordances may retain or modify ways of interacting, when there is no physical co-presence of interactants.

\section{ONLINE INTERACTION, PRIVACY MANAGEMENT, AND SOCIAL ETIQUETTE}

As communication mediums evolve, users face new opportunities and challenges when they interact or disclose information online. Among those, self-violation of privacy, or excessive disclosure, is a well-documented phenomenon (Debatin et al. 2009; Lewis, 
Kaufman, and Christakis 2008; Waters and Ackerman 2011). Social media situates different audiences from various social networks together, which is known as "context collapse" (Davis and Jurgenson 2014; Marwick and boyd 2011). Users may restrict access to their information by changing their privacy settings (Debatin et al. 2009; Litt 2012; Tian and Menchik 2016; Vitak 2012), but Marwick and boyd (2011) pointed out that the issue of context collapse still remains as circles of acquaintances, friends, co-workers, and family may coexist online and create tensions not normally found in $\mathrm{FtF}$ interactions. To counter context collapse, a variety of different mitigation strategies are used to control online privacy, including self-censorship, content withdrawal, and sharing different information in different spaces (Stutzman, Gross, and Acquisti 2013). Another privacy management strategy, known as the "lowest common denominator" approach, is only sharing information that is appropriate for everyone in the network (Hogan 2010; Marwick and boyd 2011).

Parallel to the discussion of new privacy management strategies is the changing of social norms that regulate interpersonal interactions and communication online (Breese-Vitelli, Kim, and Jenkins 2014; Preece 2004). Such social etiquette has been increasingly discussed in the literature in the past two decades. While social etiquette may vary across demographic groups and media platforms (Valdez, Schaar, and Ziefle 2013), certain types of cell phone etiquette (or "netiquette") have been documented among youths, including providing a timely response, viewing messages, and using linguistic signals and symbols in texting (AgoncilloQuirante 2006). Breaches of social etiquette online have been documented by Preece (2004) and includes annoying behavior such as sending jokes, typing in caps only, or the absence of 
grammar and punctuation. Such "poor etiquette" online, Preece (2004) noted, tends to be attributed to the lack of technical skills, failure to appreciate the priorities of others, potentially malicious behavior, and a lack of courtesy.

Some studies have briefly acknowledged online etiquette. Brownlie and Shaw's (2019) examination of emotional exchanges in online interactions found that, on Twitter, users disclosed distress without specific details in order to protect their privacy, and their audience offered subtle emotional support that was met with gratitude. Others also pointed out strategies that online users use to avoid loss of face, such as carefully choosing words to sustain harmonious relationships (Fossati 2014). Chen (2010), for instance, concluded that the Taiwanese use more ingratiation strategies, such as complimenting others and doing favors, in their self-presentation on blogs than Americans.

Some research has also been done on the different aspects of social etiquette online, although they have not been framed as such. For example, respect as a form of etiquette is exemplified when Facebook and Instagram users reciprocate "likes" (Au 2020). Elegance is demonstrated through the tendency of North American social networking site (SNS) users to present themselves positively online, and humility is often practiced by East Asian SNS users (Lee-Won et al. 2014).

Nevertheless, there is still little research on how the aforementioned three dimensions of etiquette are affected and mediated by the technical affordances of social media in a transitional society like contemporary China. Thus, examining social etiquette online, 
especially in the Chinese context that is characterized by rapid urbanization and large-scale internal migration, may better demonstrate how new forms of social etiquette have emerged and how these distinctive features of online interactions influence etiquette. This research gap will be addressed by examining China's most popular social media feed: WeChat Moments.

\section{AN ONLINE ACQUAINTANCE COMMUNITY ON WECHAT}

Through their technical designs, technologies can "request, demand, encourage, discourage, refuse and allow particular lines of action and social dynamics" (Davis 2020:12, see also Davis and Chouinard 2016). Technical affordances often construct users' perception of possible behaviors and actions to be carried out online (Gibson 2014; Norman 2013). For example, users' interaction afforded by the design of innocuous email, web interfaces, and Electronic Resource Management systems could drive surveillance, monitoring, and a "New Taylorism" (Head 2005) and video game players might be influenced subconsciously by the way game programmers and designers translate real-world issues into systematic relationships that can be ideologically inflected (Neff et al. 2012).

WeChat, the most popular instant messaging and social media app in China, serves as an ideal venue to examine how technical affordances mediate interpersonal interactions and online social etiquette among Chinese internet users because of its highly private, customized, culturally oriented user-experience design and frequently updated privacy setting features. From the analytical perspective proposed by Davis and Chouinard (2016), WeChat demands its users to register with a phone number and only allows logins through 
smartphones. The app requests users to send "friend requests" to others for approval to chat, view each other's previous and current posts, and interact on WeChat. Unlike Facebook, WeChat refuses public searches or displays of posts through mere searches by usernames. Users can only be located by searching for their registered phone number, user ID, QQ ID ${ }^{3}$, or personal $\mathrm{QR}$ code ${ }^{4}$, or are added through a group chat with mutual authentication as a default requirement for connecting as a contact (Shu et al. 2017). With such settings, WeChat provides a quasi-private online space for "more trusted" offline social groups to interact and share personal information. As most WeChat contacts are family and relatives, close friends, colleagues, and real-life acquaintances (Shu et al. 2017; Zhu et al. 2014), users are able to recreate an acquaintance community online.

Technology is both influenced by and influencing a society's or social group's culture (Bar et al. 2016; Hård and Jamison 2013; Jamison and Hård 2003). One of the most effective illustrations of this argument on WeChat is "Moments" (pengyou quan). WeChat Moments is a feature embedded in WeChat that allows users to interact by posting texts, photos, locations, news articles, external links, and videos, and mutually "liking" and commenting on posts. However, unlike the Facebook newsfeed, only comments and "likes" of one's contacts appear on the posts shown on one's Moments feed page, which means disclosure by "friends of friends" is not visible to the user (Figure 1).

\footnotetext{
${ }^{3}$ QQ is a popular instant messaging software service and web portal in China developed by Tencent.

${ }^{4}$ WeChat QR Codes are colorful square graphics coded with information. Each WeChat user has a unique QR code that could be shared and scanned to send them friend requests.
} 


\section{<insert Figure 1 here>}

Figure 1. Schematic of "Online Acquaintance Community"

Since any information disclosed by "strangers" (anyone not directly added to one's WeChat contact list) would not appear on one's feed, Moments is a striking reproduction of the small networks that make up an acquaintance community. Moreover, Moments replicates the patterns of everyday interactions among offline friend groups, which enhances the experiences of users and provides a greater sense of privacy while browsing (DeLuca, Brunner, and Sun 2016; Shu et al. 2017).

Moreover, users are encouraged to use carefully designed technical settings to manage their disclosures on Moments as they wish. Moments offers multiple ways, such as "block", "hide my/his post", and "tag", to customize interaction with different contacts. Say User A "blocks" User B. User B will not be able to access the private messages or past and future posts of User A. Conversely, User A cannot view any of User B's posts either. Apart from "blocking" User B, User A can also hide User B's posts. Thus, User B can still message User A, but User A will not see any future posts of User B. Moreover, if User A elects to "hide my posts," then User B will no longer see any past and future posts of User A.

Further, the feature of selective sharing on Moments is offered by the app to allow users to privately "tag" contacts into sharing lists so they can share customized information to customized groups of social networks to address "context collapse." As a result, users can customize their audience groups for each Moments posting. Audiences can be selected for 
each post without the knowledge of those excluded. With these "granular audience control techniques" (Li et al. 2018), users can customize how they behave in front of different audiences, which provides an ideal setting to explore how technical affordances influence social etiquette during online interaction.

\section{DATA AND METHOD}

This study focuses on educated urban young adults who reside in cosmopolitan cities, are born after market reforms in China, and who are thus more likely to adopt the social etiquette of an urban society. Sixty WeChat users between the ages of 18 and 34 were recruited from universities in Beijing, Shanghai, and Guangzhou through public-recruitment campus flyers, online advertisements on WeChat posts, and referrals by university staff. Of the sixty participants, 32 are female and 28 are male, with 44 undergraduates and 16 graduate students or non-faculty university staff members. A sequential interviewing approach was adopted where "each case provides an increasingly accurate understanding of the questions at hand" (Small 2009:24-25). Earlier interviews provided findings and questions that informed later interviews until little new information was found. The interview questions were modified to include increasingly refined questions on the factors and mechanisms that influence activities on WeChat. Information saturation was achieved around the 45th interview and subsequent interviews were carried out to validate our findings.

The initial interviews were open-ended and solicited details such as when they began to use WeChat and what changed in the way that they posted over time. Respondents discussed 
their own specific written statements on Moments and shared their thoughts when creating posts as well as answering general questions, such as "Whom did you have in mind when you were posting?", in addition to application-specific questions such as "Do you use the 'tag' function when posting on WeChat Moments?" The answers pointed to the intended audience and revealed the assigned meanings behind text-based posts. These interactional details allowed for a general analysis of the relationships between user perceptions of their audience, consideration of relational ties, and the information disclosed, shared, or commented on.

Initial interviews provided direction for the research focus. Later interviews included newly found topics from earlier interviews. The questions were organized around larger themes identified by previous focus group discussions and initial interviews that asked about intended audience, conflict, consideration of face, etiquette, relationships, and so on. Respondents were informed at the beginning of the interview that they could decline to answer certain questions or deny interviewers access to their Moments. All interviews were recorded with respondent consent, conducted in Mandarin Chinese, and translated into English by the authors.

Both thematic (Guest, MacQueen, and Namey 2012) and abductive analyses (Timmermans and Tavory 2012) were used for coding and data analysis. The former allows focus on themes that emerged from the interviews and three themes were found: giving face to others, presenting a positive but restrained self-image, and avoiding the spreading of "negative energy" on WeChat. They were the most frequent responses to "Why did you post this?" An abductive analysis requires the constant comparison of new data with existing 
theories. Existing concepts discussing online disclosure, such as "context collapse" (Marwick and boyd 2011) and " $n$-adic interaction" (Tian and Menchik 2016), was used to examine new data; however, while such concepts were helpful in accounting for why the Chinese disclose particular information on WeChat, they could not fully explain some of the perplexing phenomena, such as posting information under duress, and re-posting posts for appeasement purposes. These investigations led to the development of a new explanation for the online behavior of Chinese users; that is, they observe a kind of social etiquette that is similar to the etiquette of an acquaintance community on WeChat Moments.

\section{EMERGENCE OF CHINESE ONLINE CIVILITY}

The idea that users observed a set of social etiquette on WeChat emerged very late in the project. During the interviews, we asked respondents why they posted certain information, commented, or "liked" particular posts by others. They often replied, "It's not proper if I don't." When probed further, some respondents directed our attention to the presence of WeChat etiquette. Some even searched online and showed us different versions of WeChat etiquette on the internet. They followed these rules, especially after encounters that alerted them to the negative consequences of disregarding these rules.

A popular version of the "10 Social Etiquette Rules on WeChat" reads as follows:

1. Whoever sends a friend request should also initiate a conversation.

\footnotetext{
${ }^{5}$ See https://k.sina.cn/article_2490325853 946f5b5d01900e88i.html.
} 
2. Don't ask someone on WeChat if they are currently free or available to talk just before you send a text message. Get straight to the point.

3. Send text instead of voice messages because sending too many voice messages is impolite and inconsiderate.

4. Carefully choose your words, emojis, and stickers. Avoid those that might have sarcastic connotations.

5. Use "Okay" or "Okie" instead of "ok" or "kk" to show politeness and friendliness.

6. Sending multiple emojis or stickers is a polite way to end a conversation.

7. Don’t post on WeChat Moments before responding to your private messages.

8. Don't selectively reply comments under your WeChat Moments post if the comments received were written by contacts on the same network. Reply to everyone.

9. Don't post continuously on WeChat Moments in a short amount of time because your posts will rudely take a lot of space when your contacts browse Moments.

10. Don't forward strongly opinionated views, including sensitive matters that involve political topics and value judgments.

Rules 1 to 8 are about the rule of "respect" when interacting with others on WeChat. They give directions on who should take initiative in sending messages, how to use emojis, and polite ways to end conversations. Rule 9 is about the rule of "elegance", as it suggests that one should not post excessively as that is a self-indulgent act. Similar rules are found in 
other versions, such as "Don't post things that are too private on Moments. People may not want to know that much about you." ${ }^{\circ}$ Rule 10 is interesting as it suggests refraining from posting about sensitive topics. We will discuss why this rule constitutes another important etiquette, the rule of "tidiness", in the analysis of our findings in this paper.

During the interviews, respondents discussed their posting, commenting, and "liking" activities, and considerations in deciding how to act online. It turned out that participants showed slightly different posting habits depending on their stage of life. For example, undergraduates were generally more active in posting and interacting with their peers on Moments since they had more homogenous social networks of peers with whom they share similar topics of discussion and contacts than graduates and those in the workforce. However, more similarities than differences were found across the age groups. For example, most undergraduates added their professors or tutors on WeChat to show respect and group affiliation when interacting with them on Moments, just as graduates and those in the workplace added their superiors to convey the same sentiments. Furthermore, the face considerations and online etiquette of the undergraduates tended to largely align with those in the workforce when they accepted an internship or became involved in a student society. Therefore, the reasons behind the variations are less about life stages and more about the structures of social networks and various roles or positions adopted in particular networks, which substantially alter face considerations and social etiquette online.

\footnotetext{
${ }^{6}$ See https://language.chinadaily.com.cn/2018-03/18/content_35872332.htm.
} 
In the following sections, we will discuss the motivations behind online etiquette and behaviors as they relate to the three elements of social etiquette: respect, elegance, and tidiness.

\section{Respect: Honoring the Face of Others}

Showing respect to others is the most important etiquette rule as demonstrated by the WeChat rules above as well as the respondents in this study. In the Chinese context, showing respect to others amounts to "giving face" or "honoring face." Since participants are socially connected in small groups on Moments and can view each other's comments under a particular post, users honor the face of others by avoiding conflict, showing allegiance, and validating group membership by commenting and reposting, which also exchanges renqing. For instance, a respondent showed appreciation to her mother with the following post:

Grandma came to visit and brought so many delicious foods from my hometown. It felt like it was Chinese New Year. Nini [respondent's daughter] is thrilled. (posted along with pictures of food; Interview 53, Guangzhou, 30, female)

The respondent posted her appreciation rather than telling her mother in person because,

When I thank her in person, she is the only person who will know. But if I post it on Moments, other people can also see it. It gives her face that way. (Interview 53, Guangzhou, 30, female) 
Expressing gratitude on one's Moments is a common practice and, as noted in many interviews, it is considered more effective than saying "thank you" FtF because a show of respect online is broadcasted to a wider audience in the same social network.

Similarly, showing respect by commenting or liking posts could be appreciated as a facehonoring practice that happens more frequently and with more convenience than in $\mathrm{FtF}$ interactions. In fact, WeChat etiquette encourages users to "be a friendly audience to post on Moments, and to 'Like' or comment to compliment those who posted."' One of the interviewees, who was a research graduate student, indicated that he would even randomly "like" posts to show respect to others,

There was one time when I posted something that I thought was interesting to Moments, and yet few people liked my post. I was quite disappointed and realized that maybe everyone expects others to like their posts just like I do. Since then, I started to "like" others' posts. Often, I just keep clicking "like" for each post in my feed until I'm exhausted. I don't look at the content or who posted it. I just ... want them to know that I'm following their updates. (Interview 39, Beijing, 23, male)

Goffman (1956:10) has defined a "sincere" performer as someone "who believe[s] in the impression fostered by their performance", and a "cynical" performer as someone who has "no belief in his own act and no ultimate concern with the beliefs of his audience". In the

\footnotetext{
${ }^{7}$ See http://www.xinhuanet.com/info/2017-05/07/c 136262298.htm.
} 
case described above, the participant was very "sincere" in believing that his "likes" would show that he cared about his contacts on WeChat and help him cultivate better social relationships among his networks. However, "liking" each and every post in his feed indicates a "cynical" element in his practice, since he did not care about "the content or who posted it". In that sense, the function of "like" helps users practice the norms of respect and giving face to others in this online acquaintance community. At the same time, practicing the norms of face-giving also encourages an "insincere" aspect of their performance.

Since liking a post is often interpreted as a face-giving practice, some respondents could also be quite sensitive and selective when "liking" or commenting on posts in certain contexts. Many respondents reported that before deciding on whether to "like" a post, they tended to carefully consider their relationships with the poster and mutual friends who can access their interactions:

Liking and commenting on a post is like approaching and talking to someone in a public place. It would be really embarrassing if the person doesn't want to engage and interact with you, and simply ignores you. And if that's the case, it could be interpreted as impolite and inconveniencing the person whose post you liked or commented on. (Interview 13, Shanghai, 20, female)

The participant, who was a Year 3 undergraduate student at the time of the interview, readily associated the act of interacting with others through postings on WeChat Moments to potential feelings of embarrassment and impoliteness because she saw the act as 
"approaching someone in a public place". This shows that the participant was fully aware of the existence of the offline social networks shared with the individual concerned and took them into consideration before initiating contact. This behavior might seem contradictory in light of the research graduate student of Interview 39, but in the context of Interview 13, this undergraduate was very aware of the large, homogeneous offline network of "common friends" on Moments (mostly her fellow undergraduate students). Her social group could access the contents of any of her online interactions with others in the same social network after it had taken place on Moments.

This case thus suggests that the accessibility and visibility of interactions on WeChat Moments could sometimes make liking and commenting on posts not just accepted mutual interpersonal acts, but also performances where the impressions of mutual friends matter. For example, one respondent who was very concerned about the opinions of those "common friends" shared to us why "liking" needed to be carefully considered:

Some of my college classmates are married and some still single ... I'm married but don't have children. Some of my friends with children post about mothering, how to get pregnant, or the high costs of daycare... It feels like I'm taking sides if I like the posts of one of the groups on certain topics... They're all from the same group of friends and can see each other's posts and my interactions with them... So, I have resorted to private chat since I don't want to appear to be taking sides and openly identifying with one group over the other. (Interview 15, Shanghai, 24, female) 
Evidently, the replication of a community of acquaintances on Moments, which is made possible through the technical design, has intensified the fear of acting inappropriately and undermining offline social relationships. In this case, the mediated venue for interactions complicated defining the audience and the "front stage." Complicating things further, users may sometimes also have to create "multiple front stages" as they simultaneously interact with different contacts in the same posts (Ling 2010), which increases the perceived social stress of potential embarrassment or loss of face. Thus, being cautious has become normalized, especially when interacting with others from a large group of common contacts.

Since mutual friends can see each other's comments beneath a post, another popular strategy to show respect is through positive feedback. The respondents indicated that if User A, for example, sees that User B "liked" a post by User C, it is likely that User A will infer that User B agrees with User C. If this is a frequent occurrence, User A would assume that Users $\mathrm{B}$ and $\mathrm{C}$ have a close offline relationship. However, this also means that when one person in a group "likes" or comments on a post, others feel pressured to do the same to avoid discrediting the face of the original poster.

A similar popular strategy to show respect and give face to others is to "like" a post and then repost the "liked" post on one's Moments. For example, a student respondent often "liked" his professor's posts and reposted them, especially when other fellow students did so, as a show of allegiance. This strategy communicates to the original poster, "I agree with you and you have my attention." In this sense, online posts, comments, and "likes" are used as "gifts" with the expectation that they would be reciprocated later. This is most common when 
there is a power difference between users, for example, juniors versus seniors or subordinates versus superiors in the workplace. Low-ranking individuals used this strategy of "liking and reposting" to show respect to high-ranking people but did not expect those persons to do the same (see also Tian 2020).

The respondents also tended to give face to others when there is a "renqing dilemma," that is, when someone asks for a favor, the respondent must save the face of the requesting party by either accommodating or declining them with good reason (Hwang 1987). In fact, reposting or "liking" a link for renqing purposes is very common on WeChat. The contents are usually commercial in nature, which is self-serving for the requester but aggravating to the poster. Nonetheless, many comply if they feel that their guanxi is strong enough to "give face" to the requester, even if they do not support the post itself. For example, many users would help to share certain advertising or commercial information from their Moments account by request. However, users also found that some content does not align with their idealized self-presentation or might serve as unwanted spam to other contacts that could see the repost in their Moments. In other words, when the social request of "respect" and "facehonoring" conflicts with one's desire to adhere to the social etiquette rule of "elegance," the user faces a social dilemma and finds ways to tackle the challenge.

When the above situation occurs, participants were found to take advantage of the technical affordances on Moments to carry out tactful navigating strategies. Two primary strategies are often used to reconcile the conflict between honoring the face of others and posting disclosures incompatible with their ideal self-image. 
The first strategy is to repost the content so that the requester would see that the post has been shared as a form of renqing. Usually, the requester would "like" the repost to express their gratitude and acknowledge the reception of renqing once they saw on Moments the repost of their requested content. However, as WeChat allows users to delete past posts whenever they want, the individual who reposted the information would later remove the post after it had served its renqing purpose in order to adhere to the social etiquette of "elegance" and protect their idealized self-image. In this case, users utilize "cynical" strategies tactfully to solve the problem of "renqing dilemma" while accomplishing their "sincere" intention of cultivating important interpersonal relationships.

The second strategy is to use the technical settings of Moments for selective sharing. Although selective sharing is supposed to allow users to privately "tag" contacts into sharing lists to avoid breach of privacy and unintended disclosure, the feature is found to have been tactfully used by many to perform or even fake renqing favors as a way to act "nice" and give face to the requester. Many respondents used the "tag" function to share renqing posts to a very limited audience, and sometimes, in more extreme circumstances, only shared it with the requester. Indeed, sharing a post with only one person or a small group in one's contact list while pretending to share it with everyone often involves negotiating renqing and face. Some users who reposted the content would even create a new group of contacts with the requester and share the post to this new list. The post would then only appear in the feed of the requester, although it might look like it had been widely shared to many. Many respondents indicated they have used this strategy to provide a renqing favor: 
One of my acquaintances asked me to share a blurb about his business on my Moments. He asked me several times, so I figured that I should give him some face ... I shared it but made it so that only he saw the post. (Interview 59, Shanghai, 31, male)

While this way of utilizing the selective sharing feature was not encouraged by the app's developers, it was nonetheless tactfully appropriated by users and became a widely used solution to an etiquette problem. When pushed to post information incompatible with their self-image, selective sharing allows users to both "give face" to others and protect their own. Selective sharing therefore can allow users to maintain the social harmony of interactions that is central to Chinese "relationship culture." However, just as the first strategy described earlier, this particular practice involves an element of "insincere performance" of face giving that, if discovered, would be extremely detrimental to the relationship and embarrassing to the poster. Understanding the potential risk, users think carefully about adopting the strategy, especially in light of the network ties held by the requester:

I cannot use the trick when I know the requester shares a large social network of common friends with me. For example, if my undergraduate fellow classmate asks me to share an advertising post for his student society, I cannot share it only to him, since if I do that, our common friends won't be able to see the repost and thus there would be no "likes" or "comments" under the repost, and thus he would instantly find out that I am using selective sharing and shared it 
only to him ... I would have to at least repost the content to all of our common friends in such cases. (Interview 35, Beijing, 20, male)

The quasi-private interactive space on Moments has lent visibility to the process of showing respect, creating more opportunities and motivation to conduct mutual facehonoring practices, and giving pressure to find options to balance the intricacies of showing respect while protecting elegance. Nevertheless, the etiquette surrounding respect is complicated by the distinct features of online interaction. Both the positive and negative effects of showing respect to others in public are amplified by the visibility of online disclosure and the fact that the design of Moments makes it possible for users to replicate their acquaintance community online. To avoid inappropriate self-presentations, one often sincerely considers how frequently they should respond, what content they should post, and the anticipated feelings of one's audience. However, it must also be noted that the technical affordances, once understood, adopted, and appropriated by users, could also encourage deception and insincere performances.

Elegance: Presenting an Idealized but Restrained Self-image

Elegance is the second element of social etiquette. In offline FtF interactions, elegance requires taking appearance and behavior into consideration. Elegance is about the expression of a good self-image. Claiming a better image in front of others is a universal phenomenon and research suggests that the Chinese tend to present themselves more modestly as flaunting might not only cause a loss of face but also hurt that of others (Hu 1944). However, this has 
begun to change on Chinese social media. Some researchers suggest that Chinese youth are becoming accustomed to describing and presenting themselves in more positive ways on SNSs, albeit less frequently or extensively than their American counterparts (Chen 2020; Mazur and Li 2016). Self-promotion, enhancement, and competitive self-presentation strategies have been observed in Chinese SNSs as youths seek to present more desirable selfimages to attract and build relationships with new friends (Chen 2010; Chu and Choi 2010; Zhao and Jiang 2016).

Similar to the Western context, the presentation of a positive self-image is prevalent on WeChat. For example, users are particularly keen to post about expensive purchases, career milestones, overseas travel, social gatherings, and children. Sometimes these efforts are rather obvious to their audience:

Some of my classmates who go abroad for exchange post very much about trivial stuff on Moments, things that are not even worth posting, such as eating with a bunch of people, house parties, and so on. I bet their targets were not the people at the dinner or party since most foreigners do not even use WeChat. I think they are actually posting those photos for their friends in China to see. (Interview 26, Shanghai, 25, male)

Notably, unlike the traditional acquaintance community, where everyone knows the business of everyone else, Chinese urbanites no longer reside in physical proximity to their past acquaintances, so Moments is now used to announce recent achievements as a means of gaining or enhancing face amongst multiple "circles of friends" and to consequently show 
personal elegance. Our interviews revealed that those who have recently achieved upward mobility or experienced positive changes are more likely to post content to show that they have done well, and indeed, the online acquaintance community encourages users to provide updates to offline acquaintances who reside in other locations as evident by posting behaviors:

We care more about our status and personal image in front of our friends, family, and other acquaintances than strangers. Those who can see your posts on WeChat are those you know in real life, so of course you are going to show off to them instead. (Interview 8, Beijing, 30, female)

Additionally, users often manipulate the unique privacy settings of WeChat as part of their strategy to maintain elegance:

Others cannot see the messages posted on your Moments from people who aren't their friends. So, when there are no "likes" or comments... maybe a lot of people have commented but because they're not your friends, you can't see the responses. My strategy is to comment under my own posts as if I were replying to someone... This creates an impression that there are a lot of people commenting on my post. (Interview 2, Shanghai, 20, male)

This user manipulated the technical features of WeChat to create an illusion that he was more popular than he truly was to his audience, thereby saving face and salvaging his selfimage in a way that is not possible in FtF interactions. 
Despite these technical affordances, respondents tended to disclose less information as their contacts accumulated over time to avoid being seen as inelegant. Many, especially university students, realized that it is disadvantageous to reveal certain personal information online, especially if they flaunted their lifestyle, because they could offend audiences in different social networks through acts of self-indulgence or arrogance. As contacts increase, many users started to recognize the need to intentionally post less frequently because they knew there is a greater likelihood of unexpected backlash from the enlarged audience, and as a result, they chose to conform to the etiquette of an acquaintance community:

I changed how I posted on Moments as I started to add more friends. I had to think more carefully before posting. I have a lot of different friends and can't be bothered to tag them in groups for selective sharing, so [...] I consider the potential impact on my relationships with others before I say anything. (Interview 5, Beijing, 25, male)

Many respondents found that increased contact diversity made it more challenging to speak their mind and compelled them to change their posting frequency. This is because different groups have different expectations of themselves and it is difficult for the ego to maintain elegance with only one presentation of self. In this regard, the problems encountered by Chinese WeChat users are similar to the "context collapse" identified by other researchers. For example, Oolo and Siibak (2013) found that Western social media users create groups of "ideal audiences" on their social media accounts, presenting to the strongest or most valued audience (Marder et al. 2016) or assuming the "lowest common denominator" approach 
where only information appropriate for everyone in the network is shared (Hogan 2010). However, to Chinese WeChat users, the decision on whether or not to adopt the "ideal audiences" or "lowest common denominator" approach is based on considering face in various social networks. To maintain elegance based on etiquette rules of showing modesty, sociability, and amicability, they often self-consciously regulate their online posting behavior to refrain from posting certain content or disclosing to a smaller or wider audience to show group affiliation, give face to others, and sometimes, post something for someone for the sake of posting something.

One indicator of the complexity of managing elegance is that respondents often realized that they had to balance how much and how often they should disclose on Moments. On one hand, excessive posting of private and intimate life details, although not disagreeable, might be inappropriate because it is seen as excessive self-indulgence. Our respondents denounced users who flooded their Moments with photos of their romantic relationships, pets, babies, selfies, or information that only the poster would appreciate. Instead of viewing such selfdisclosure as positive expressions of values and presentations of self-image, they were seen as self-indulgent and negligent of the feelings of others because these postings were "irrelevant" to the majority of contacts. These types of posts could therefore be made private or topics with fewer "likes" and comments could be lessened as a way to maintain elegance. However, the poster would need to have a sense of self-awareness too.

On the other hand, users also expressed that they cannot stop engaging on WeChat altogether because if nothing is posted, they risk being seen as defensive, unfriendly, or 
disrespectful. As one's past posts on WeChat Moments are usually permanent and visible to every contact in the personal "album" section, posting is not only a performance, but also an exhibition and archive of the self (Zhao et al. 2013), and therefore, "not posting" could be equated to "unwilling to share" or "keeping to oneself." A lack of engagement or long lapses between posts also carries the risk that others might believe they have been intentionally blocked. As an interviewee shared, "To stop sharing information is a sign that the interpersonal relationship is no longer friendly, which will have a very negative impact on the offline relationship" (Interview 48, Guangzhou, 25, male). Therefore, instead of hiding posts from others or blocking other users, some users maintain the elegance of social harmony by reminding themselves to post superficial content sporadically, and others use the aforementioned "tag" feature to selectively share posts. Respondents used these strategies so as not to appear "strange," "unfriendly," or labeled as "someone who does not post."

As the above analysis shows, users consider a variety of factors and utilize technical affordances of WeChat design in order to adhere to the etiquette of maintaining elegance online. It could be inferred that though users share some basic norms of what is acceptable and civil and what constitutes "good etiquette" on WeChat, those requirements can contradict. In tackling conflicting requirements from different aspects of etiquette rules, users often have to decide whether to reveal or conceal, and to post to a wider or narrower audience, depending on the technical affordances available to them, the specific situation, and their consideration of particular relationships among the groups of audiences who could access the post in their networks. A positive self-image following the rules of "elegance" has 
thus become more adjustable and tactful but also more detailed-oriented and time-consuming when preserved and maintained online.

\section{Tidiness: Do Not Spread "Negative Energy"}

Just as in FtF interactions, how one's behavior influences the "tidiness" of the interacting environment is also applicable to the online space. Thus, the last item on the WeChat social etiquette rules list—do not share strong opinions, especially sensitive matters that involve political topics and value judgments - is important. Inappropriate self-disclosure and revealing one's "true self" can cause uneasiness, provoke criticism, and thereby bring about "negative energy." The rule of "tidiness" is different from what Goffman (1963) coined "tactful inattention" or "civil inattention," which refers to the mutual expectation that an outsider would avert their eyes from other's unintended disclosures to collectively protect others" bounded backstages (Armond 2018). Instead, the rule of "tidiness" creates and increases the social expectation to only disseminate "appropriate," "decent" and "positive" information to everyone in the community, while at the same time, it encourages selfcensorship and inhibits posting provocative content because Moments contacts usually include colleagues or clients who are not friends by choice and are possibly not like-minded. Nevertheless, these contacts are always present and can readily access posts, which subject users to the gaze of their larger social circle. To explicitly support or oppose a view or value can be professionally or personally harmful to cultivating interpersonal relationships and developing social networks. 
Very much like what Lau and Wong (2008) found, tidiness in the online interacting environment concerned social harmony and stability; thus, WeChat users in this study tended to encourage socially sanctioned expressions and discourage assertive action:

WeChat Moments is used to appear sociable. You can't be too eccentric, otherwise people will think that you're being difficult. If you're always talking about issues like air pollution and the environment, people will wonder about you and think you're strange. (Interview 18, Guangzhou, 19, female)

Soon realizing that posting about air pollution is not socially acceptable, this respondent changed her posting behavior to share photos on food and travel, just like everyone else.

The users also tried to avoid posting personal opinions or views that could potentially lead to conflict. Disclosed information or posted responses were rarely genuine:

We really should not post our opinions... It would be humiliating and embarrassing if there were conflicting opinions. You might damage your relationship with others. (Interview 36, Shanghai, 20, female)

Many respondents felt that posts should be positive and upbeat because it is important to appear congenial, especially in the workplace:

You can do whatever you want behind closed doors at home. However, if you want to survive in the workplace, you need people to help you, [so] you have to appear agreeable. (Interview 12, Shanghai, 26, female) 
I never post negative things, even if I might have something I really want to say, because happy things are more palatable on WeChat. (Interview 25, Shanghai, 23 , female)

Many respondents emphasized the importance of "not spreading negative energy" on WeChat because that content could cause disputes, or discomfort in others, thus "polluting the online public space." "Negative energy" is a broad and vague term. It can include recent events that reflect the darker side of society (such as food safety, child abuse, and sexual harassment), negative emotions (such as depression or hopelessness), complaints, rumors, or sensationalized events. By contrast, "positive energy" refers to upbeat and positive posts, such as those expressing gratitude or appreciation.

While users are often pressured to occasionally disclose on Moments to adhere to elegance, "tidiness" means that certain content is generally unwelcome on Moments, even though they may be sincere opinions. Keeping those rules in mind, many users did not share authentic views and tended to produce shallow and meaningless posts, which signals cynicism and insincerity:

Having in-depth discussions and developing meaningful relationships must be done privately ... The things we post on Moments are usually insignificant and we don't speak our mind. (Interview 44, Beijing, 21, female)

Honest opinions or serious dialogue can be found on Weibo (a Chinese social media similar to Twitter) where people engage in fierce dialogue and fight aggressively. Since the 
audience on Weibo mainly comprises anonymous strangers (Deluca et al. 2016), they do not need to maintain interpersonal harmony or follow the social etiquette of an acquaintance community:

On Weibo, you can say anything [...] You can talk more freely to strangers. (Interview 45, Beijing, 34, male)

Since online etiquette on Weibo does not follow an acquaintance community, there is no pressure to follow rules that pertain to respect or elegance within an audience of strangers. As a result, there is almost no consensus on Weibo, let alone the maintenance of harmonious relationships (Lin and Tian 2019). In fact, one version of WeChat etiquette notes this difference between WeChat and Weibo and suggests, "Do not argue or pick a fight with your contacts on WeChat Moments. If you really want to vent, move over to Weibo. It's better to stay friendly and humble on WeChat.",

Interpersonal harmony and maintaining guanxi carry much weight on WeChat and there is no question around the dance of civility. While WeChat replicates the offline world by bringing FtF relationships online, there are more repercussions in the online sphere because the audiences are larger, and posts are traceable. As a result, WeChat posts are generally noncontroversial in nature, mainstream values are reinforced, and posted content is safe for mass consumption. The prevalence of confirmative and self-censored content is normalized and has superseded other forms of sincere and straight-forward self-expressions on Moments.

\footnotetext{
${ }^{8}$ See http://www.xinhuanet.com/info/2017-05/07/c 136262298.htm.
} 
These findings on practices around showing respect to others, proper self-presentation, and maintaining one's tidiness online suggest that a new set of etiquette rules have emerged as criteria of civil practices and become observed normative behaviors on WeChat. Although these rules are sometimes in conflict with each other, at the heart of these rules is the sincere motivation to maintain interpersonal harmony, which is facilitated by technical designs of a mediated environment; thus, rules become internalized and normalized, and users are mindful of the expectations to perform and maintain social etiquette online.

\section{DISCUSSION AND CONCLUSION}

This paper argues that the rapid modernization and urbanization of China over the past four decades meant that offline, Chinese urbanites have been adapting to interactional norms found in a stranger society, but the traditional social elements of an acquaintance community are found to be preserved by internalized social etiquette and social practices, such as respect, elegance, and tidiness, as suggested by Zhao (2016), on WeChat. In terms of the etiquette around respect, WeChat users tend to honor the face of others and avoid causing others to lose face. In terms of the etiquette around elegance, they try to present a positive self-image but also refrain from excessive flaunting. As for the etiquette around tidiness, they preserve the cleanliness of the online public space by avoiding negativity. Through adhering to etiquettes of "respect", "elegance" and "tidiness", users accomplish their sincere intentions to sustain important and meaningful offline relationships, although the actual practice may be carried out with cynical and contradictory elements facilitated by technical affordances of the platform. 
Since a vast majority of WeChat contacts are also offline acquaintances, relationships between online contacts on WeChat are expected to have longevity and extend into the offline world. The unique technical layout of Moments has also resulted in a communication environment that replicates offline small-group interactions. This particular context, together with the closed and private settings for information sharing on WeChat, results in users' adherence to the social etiquette of an acquaintance society. Users prioritize managing offline relationships online and accommodating the needs of others to show respect. Maintaining interpersonal harmony is given precedence and discrediting the face of others is avoided. Therefore, the avoidance of negativity cannot be entirely attributed to state censorship because even if disclosures were not politically sensitive, they were scrutinized by other users. Nevertheless, users seriously contemplate how posts would be interpreted and the subsequent impact on their relationships. Contradictory practices could sometimes also be observed on Moments as the result of specific contexts, the envisioning of one's own social network structures, and considerations about different social etiquette rules by users. Thus, WeChat Moments has not only increased the challenge of renouncing the cultural norms of interpersonal harmony, it has also given way to new observed norms of virtual civility through the use of digital technology.

The main contributions of this paper lie in two areas. First, WeChat is the most popular social media platform in China, yet little is known about how and why people interact on WeChat. Previous studies have focused on SNSs such as Twitter and Facebook. However, Moments differs in its unique technological design as a quasi-private community 
and its feature set allows "granular audience control techniques" (Li et al. 2018) so users can maximize communication control. Thus, this study addresses the knowledge gap by offering an in-depth qualitative account of how Chinese users use technical tools while partially explaining how Moments integrates with Chinese interactional culture.

Second, this study examines online social etiquette and finds that it is ingrained in the structure of the community and intensified by the technical affordances and mediated online interactions. That is, when offline interpersonal relationships and interactions are enacted and mediated online, the social etiquette rules and traditional norms of an acquaintance society could be preserved through specific technical designs. For example, this paper illustrates and contextualizes what and how WeChat may "request, demand, encourage, discourage, refuse and allow" of its users (Davis 2020; Davis and Chouinard 2016), such as the removal of disclosures posted by "strangers" and selective sharing, thus encouraging adherence to the etiquette rules of Chinese civility. Furthermore, it has also been shown that the distinct features of online interaction, such as recordability and accessibility of past interactions, and an undetectable audience (Tian 2020) can sometimes increase the social stress of virtual interactions, making users proceed with care. As a result, many Chinese users actively abide with etiquette rules on $\mathrm{WeChat}$ more stringently than in actual $\mathrm{FtF}$ situations to sincerely sustain meaningful offline social relationships, and may develop insincere and cynical performances online to show conformity to connections in one's offline social networks.

\section{ACKNOWLEDGMENTS}

This work was supported by the General Research Fund (GRF), Research Grants Council, 
HKSAR (project number: 17662416). We thank the two anonymous reviewers and the editor of the journal for the helpful comments.

\section{REFERENCES}

Agoncillo-Quirante, Aleli. 2006. “Texting, Etiquette and Social Relationships in Teens' Interpretive Communities.” Pp. 1-15 in Mobile and Popular Culture: Asia Culture Forum, Session 2: Citeseer.

Au, Anson. 2020. "Guanxi 2.0: The Exchange of Likes in Social Networking Sites.” Information, Communication and Society 23(13):1-16.

Bar, François, Matthew S. Weber, and Francis Pisani. 2016. "Mobile Technology Appropriation in A Distant Mirror: Baroquization, Creolization, and Cannibalism.” New Media and Society 18(4):617-636.

Barley, Stephen. 1986. “Technology as an Occasion for Structuring: Evidence from Observations of CT Scanners and The Social Order of Radiology Departments." Administrative Science Quarterly 31(1):78-108.

Bian, Yanjie. 1994. "Guanxi and the Allocation of Urban Jobs in China." The China Quarterly 140:971-99.

Breese-Vitelli, Jennifer, Philip Kim, and Kay Jenkins. 2014. “Text Messaging Etiquette: An Exploration of Social Breaches." Issues in Information Systems 15(2):157-167. 
Brown, Penelope and Stephen Levinson. 1987. Politeness: Some Universals in Language Usage. New York: Cambridge University Press.

Brownlie, Julie and Frances Shaw. 2019. "Empathy Rituals: Small Conversations about Emotional Distress on Twitter.” Sociology 53(1):104-122.

Burt, Ronald, Yanjie Bian, Lijun Song, and Nan Lin, eds. 2019. Social Capital, Social Support and Stratification: An Analysis of the Sociology of Nan Lin. Cheltenham, Gloucestershire: Edward Elgar Publishing.

Burt, Ronald and Katarzyna Burzynska. 2017. "Chinese Entrepreneurs, Social Networks, and Guanxi." Management and Organization Review 13(2):221-260.

Chen, Baifeng. 2019. Ban Shuren Shehui: Zhuanxing Qi Xiangcun Shehui Xingzhi Shenmiao [the Quasi-Acquaintance Society: A Thick Description of the Rural Society in Transition]. Beijing: Social Sciences Academic Press.

Chen, Yi-Ning Katherine. 2010. "Examining the Presentation of Self in Popular Blogs: A Cultural Perspective." Chinese Journal of Communication 3(1):28-41.

China Internet Network Information Center (CNNIC). 2016. 2016 Chinese Social Media User Behaviors Report. Retrieved Jun 17, 2020 (http://www.cnnic.net.cn/hlwfzyj/hlwxzbg/sqbg/201712/P020180103485975797840.pd f). 
China Internet Network Information Center (CNNIC). 2018. The $41^{\text {th }}$ Report on The Development of Internet in China. Retrieved Jan 18, 2019

(http://www.caict.ac.cn/kxyj/qwfb/ztbg/201805/P020180529380481819634.pdf).

Chu, Shu-Chuan and Sejung Marina Choi. 2010. "Social Capital and Self-Presentation on Social Networking Sites: A Comparative Study of Chinese and American Young Generations." Chinese Journal of Communication 3(4):402-420.

Davis, Jenny. 2020. How Artifacts Afford: The Power and Politics of Everyday Things. Cambridge, Massachusetts: MIT Press.

Davis, Jenny and James Chouinard. 2016. "Theorizing Affordances: From Request to Refuse.” Bulletin of Science, Technology and Society 36(4):241-248.

Davis, Jenny and Nathan Jurgenson. 2014. "Context Collapse: Theorizing Context Collusions and Collisions." Information, Communication and Society 17(4):476-485.

De Armond, Elizabeth. 2018. "Tactful Inattention: Erving Goffman, Privacy in The Digital Age, and the Virtue of Averting One's Eyes.” John's Law Review 92(2):283-325.

Debatin, Bernhard, Jennette Lovejoy, Ann-Kathrin Horn, and Brittany Hughes. 2009. "Facebook and Online Privacy: Attitudes, Behaviors, and Unintended Consequences.” Journal of Computer-Mediated Communication 15(1):83-108. 
DeLuca, Kevin Michael., Elizabeth Brunner, and Ye Sun. 2016. “Constructing Public Space:

Weibo, Wechat, and the Transformative Events of Environmental Activism in China.” International Journal of Communication 10:321-339.

Elias, Norbert. 1978. The History of Manners (E. Jephcott, Trans.). New York: Pantheon Books (Original work published 1939).

Fei, Hsiao-Tong. 1948. Peasant life in China. London: Routledge and Kegan.

Fei, Hsiao-Tong. 1992. From the Soil, the Foundations of Chinese Society: A Translation of Fei Xiaotong's Xiangtu Zhongguo (G. G. Hamilton and W. Zheng, Trans.). Berkeley and Los Angeles, CA: University of California Press (Original work published 1947).

Fossati, Serena. 2014. "Identity Construction Online: A Study of the Chinese Social Networking Site Renren.” Comunicazioni Sociali 2:336-346.

Fried, Morton. 1953. Fabric of Chinese Society: A Study of The Social Life of a Chinese County Seat. New York: Frederick A. Praeger, Inc.

Gibson, James. 2014. The Ecological Approach to Visual Perception: Classic Edition. Hove, UK: Psychology Press.

Goffman, Erving. 1955. “On Face-Work: An Analysis of Ritual Elements in Social Interaction.” Psychiatry 18(3):213-231.

Goffman, Erving. 1956. The Presentation of Self in Everyday Life. Edinburgh, UK: University of Edinburgh Social Sciences Research Center. 
Goffman, Erving. 1963. Behavior in Public Relation: Notes on The Social Organization of Gatherings. Macmillan.

Goffman, Erving. 1967. Interaction Ritual: Essays in Face-to-face Behavior. New York: Doubleday.

Guest, Greg, Kathleen M. MacQueen, and Emily E. Namey. 2012. Applied Thematic Analysis. Thousand Oaks, CA: Sage.

Han, Changfu. 2006. "The Trend and Perspective of Rural Migrant Workers in China.” Economic Research Journal 2006(12):4-12.

Hård, Mikael and Andrew Jamison. 2013. Hubris and Hybrids: A Cultural History of Technology and Science. London: Routledge.

Head, Simon. 2005. The New Ruthless Economy: Work and Power in The Digital Age. New York: Oxford University Press.

Hogan, Bernie. 2010. "The Presentation of Self in The Age of Social Media: Distinguishing Performances and Exhibitions Online.” Bulletin of Science, Technology and Society 30 (6):377-386.

Hsu, Francis L. K. 1953. Americans and Chinese: Two Ways of Life. New York: Henry Schuman.

Hsu, Stephen Chuanhsi. 1996. "Face”: An Ethnographic Study of Chinese Social Behavior. New Haven, CT: Yale University Press. 
Hwang, Kwang-kuo. 1987. "Face and Favour: The Chinese Power Game.” American Journal of Sociology 92(4):944-74.

Jamison, Andrew and Mikael Hård. 2003. "The Story-Lines of Technological Change: Innovation, Construction and Appropriation." Technology Analysis and Strategic Management 15(1):81-91.

Jiang, Zhaocai and Xiuxin Zhao. 2016. "Self-Control and Problematic Mobile Phone Use in Chinese College Students: The Mediating Role of Mobile Phone Use Patterns.” BMC Psychiatry 16(1):Article 416.

Jiao, Yu-liang. 2015. “Acquaintance Society, Stranger Society, and the Market Trading Order: A Discussion with Professor Liu Shao-jie." Sociological Review of China 3(3):25-32.

Kelly, Kevin. 2010. What Technology Wants. New York: Penguin.

Kinnison, Li Qing. 2017. "Power, Integrity, and Mask - an Attempt to Disentangle the Chinese Face Concept.” Journal of Pragmatics 114:32-48.

Lau, Ying and Daniel Fu Keung Wong. 2008. "Are Concern for Face and Willingness to Seek Help Correlated to Early Postnatal Depressive Symptoms Among Hong Kong Chinese Women? A Cross-Sectional Questionnaire Survey.” International Journal of Nursing Studies 45(1):51-64.

Lee-Won, Roselyn, Minsun Shim, Yeon Kyoung Joo, and Sung Gwan Park. 2014. "Who Puts the Best 'Face' Forward on Facebook? Positive Self-Presentation in Online Social 
Networking and the Role of Self-Consciousness, Actual-To-Total Friends Ratio, And Culture." Computers in Human Behavior 39:413-423.

Lewis, Kevin, Jason Kaufman, and Nicholas Christakis. 2008. "The Taste for Privacy: An Analysis of College Student Privacy Settings in An Online Social Network." Journal of Computer-Mediated Communication 14(1):79-100.

Li, Yao, Xinning Gui, Yunan Chen, Heng Xu, and Alfred Kobsa. 2018. "When SNS Privacy Settings Become Granular: Investigating Users' Choices, Rationales, and Influences on Their Social Experience." Proceedings of the ACM on Human-Computer Interaction $2(C S C W): 1-21$.

Lin, Nan, Yang-chih Fu, and Chih-Jou Jay Chen, eds. 2013. Social capital and its institutional contingency: A study of the United States, China and Taiwan. London: Routledge.

Lin, Tony Zhiyang and Xiaoli Tian. 2019. “Audience Design and Context Discrepancy: How Online Debates Lead to Opinion Polarization.” Symbolic Interaction 42(1):70-97.

Ling, Richard. 2010. New Tech, New Ties: How Mobile Communication is Reshaping Social Cohesion. Cambridge, US: MIT press.

Litt, Eden. 2012. “Knock, Knock. Who's There? The Imagined Audience.” Journal of Broadcasting and Electronic Media 56(3):330-345.

Liu, Shaojie. 2002. “Zhongguo Shehui Zhuanxing Zhong De Ganxing Xuanze.” Jiangsu Social Sciences 2002(02):17-21. 
Liu, Shaojie. 2014a. "Mosheng Guanxi Shuxi Hua: Youhua Shichang Jiaoyi Zhixu De Bentuhua Xuanze.” Fujian Tribune (Social Sciences Edition) 2014(04):160-167.

Liu, Shaojie. 2014b. "The Social Foundation of Chinese Market Order: A Concurrent Comment on the Argument that Chinese Society is Acquaintance Society or Stranger Society." Sociological Review of China 2014(02):28-342.

Ma, Liangcan. 2015. "How to Maintain the Market Transaction Order: Discuss with Jiao Yuliang." Sociological Review of China 2015(05):88-96.

Marder, Ben, Adam Joinson, Avi Shankar, and Kate Thirlaway. 2016. "Strength Matters: SelfPresentation to the Strongest Audience rather than Lowest Common Denominator when Faced with Multiple Audiences in Social Network Sites." Computers in Human Behavior 61:56-62.

Marwick, Alice and Danah boyd. 2011. "I Tweet Honestly, I Tweet Passionately: Twitter Users, Context Collapse, and the Imagined Audience." New Media and Society 13(1):114-133.

Mazur, Elizabeth and Yidi Li. 2016. "Identity and Self-Presentation on Social Networking Web Sites: A Comparison of Online Profiles of Chinese and American Emerging Adults." Psychology of Popular Media Culture 5(2):101-118.

Neff, Gina, Tim Jordan, Joshua Mcveigh-Schultz, and Tarleton Gillespie. 2012. "Affordances, Technical Agency, and the Politics of Technologies of Cultural Production." Journal of Broadcasting and Electronic Media 56(2):299-313. 
Norman, Don. 2013. The Design of Everyday Things: Revised and Expanded Edition. New York: Basic Books.

Oolo, Egle and Andra Siibak. 2013. "Performing for One's Imagined Audience: Social Steganography and Other Privacy Strategies of Estonian Teens on Networked Publics.” Cyberpsychology: Journal of Psychosocial Research on Cyberspace 7(1):Article 7.

Pan, Yuling. 2000. Politeness in Chinese Face-To-Face Interaction (No. 67). Greenwood Publishing Group.

Papa, Wendy H. and Michael J. Papa. 1992. "Communication Network Patterns and the ReInvention of New Technology." The Journal of Business Communication 29 (1):41-61.

Preece, Jenny. 2004. "Etiquette Online: From Nice to Necessary." Communications of the ACM 47(4):56-61.

Reader, Bill. 2012. "Free Press Vs. Free Speech? The Rhetoric of "Civility" in Regard to Anonymous Online Comments.” Journalism and mass communication quarterly 89(3):495-513.

Shu, Cong, $\mathrm{Na} \mathrm{Hu}$, Xing Zhang, Yuanxiao Ma, and Xu Chen. 2017. “Adult Attachment and Profile Images on Chinese Social Networking Sites: A Comparative Analysis of Sina Weibo and Wechat." Computers in Human Behavior 77:266-273.

Simmel, Georg. 1922. "The Web of Group Affiliations (Reinhard Bendix, Trans.)." Conflict (Kurt H. Wolff, Trans.). New York: Free Press of Glencoe. 
Simmel, Georg. 1950. The Sociology of Georg Simmel. New York: The Free Press.

Small, Mario Louis. 2009. "How Many Cases Do I Need? On Science and the Logic of Case Selection in Field Based Research.” Ethnography 10(1):5-38.

Stutzman, Frederic, Ralph Gross, and Alessandro Acquisti. 2013. “Silent Listeners: The Evolution of Privacy and Disclosure on Facebook." Journal of Privacy and Confidentiality 4(2):7-41.

Tencent. 2020. 2020 Annual Report. Retrieved Jun 17, 2020 (https://cdc-tencent-com1258344706.image.myqcloud.com/uploads/2020/04/02/ed18b0a8465d8bb733e338a1a be76b73.pdf)

Tian, Xiaoli. 2016. "Network Domains in Social Networking Sites: Expectations, Meanings, and Social Capital.” Information, Communication and Society 19(2):188-202.

Tian, Xiaoli. 2020. “An Interactional Space of Permanent Observability: Wechat and Reinforcing the Power Hierarchy in Chinese Workplaces.” Sociological Forum: Online $\begin{array}{llll}\text { First. } & \text { Retrieved } & \text { December } & 3,\end{array}$ (https://onlinelibrary.wiley.com/doi/10.1111/socf.12662)

Tian, Xiaoli and Daniel A. Menchik. 2016. “On Violating One's Own Privacy: N-adic Utterances and Inadvertent Disclosures in Online Venues." Studies in Media and Communications 11:3-30.

Timmermans, Stefan and Iddo Tavory. 2012. "Theory Construction in Qualitative Research from Grounded Theory to Abductive Analysis.” Sociological Theory 30(3):167-186. 
Valdez, André Calero, Anne Kathrin Schaar and Martina Ziefle. 2013. "Personality Influences on Etiquette Requirements for Social Media in the Work Context." Pp. 427-46 in International Conference on Human Factors in Computing and Informatics: Springer.

Venkatesh, Viswanath, James YL Thong, and Xin Xu. 2012. "Consumer Acceptance and Use of Information Technology: Extending the Unified Theory of Acceptance and Use of Technology." MIS Quarterly 36(1):157-178.

Vitak, Jessica. 2012. “The Impact of Context Collapse and Privacy on Social Network Site Disclosures.” Journal of Broadcasting and Electronic Media 56(4):451-470.

Wang, Mingming. 2006. "Paradigm and Transcendence: An Anthropological Study of the Chinese Society." Journal of Guangxi University for Nationalities (Philosophy and Social Sciences Edition) 28(4):67-74.

Wang, Hongchang, Xuehua Liao, and Jinghua Xiao. 2014. "Legitimacy Dilemma of Chinese Guanxi Theory: A Perspective of Social Norms." Chinese Journal of Management 11(5):764-771.

Waters, Susan and James Ackerman. 2011. "Exploring Privacy Management on Facebook: Motivations and Perceived Consequences of Voluntary Disclosure." Journal of Computer-Mediated Communication 17(1):101-115.

Wu, Chongqing. 2011. "From the Society of Acquaintances to the Subjectless Society of Acquaintances.” Dushu 2011(01):19-25.

Yan, Yunxiang. 2017. "Civility, Taiwanese Civility, and the Taiwanese Civility Reconstructed by Mainland Chinese.” Pp. 233-257 in Taiwan's Impact on China: Why Soft Power 
Matters More than Economic or Political Inputs, edited by Steve Tsang. The Nottingham China Policy Institute Series. Cham, Switzerland: Springer International Publishing.

Zhai, Xuewei. 2004. “Renqing, Mianzi, Yu Quanli De Zai Shengchan: Qingli Shehui Zhong De Shehui Jiaohuan Fangshi [Favor, Face and Reproduction of the Power: A Way of Social Exchange in an Reasonableness Society].” Sociological Research 5(4):48-57.

Zhao, Shanyang. 2016. “Zhong Mei Li Yi Cha Yi De Ren Ji Guan Xi Fen Xi [Analysis of SinoU.S. Differences in Social Etiquette from the Standpoint of Self-other Relations].” Journal of Social Science of Hunan Normal University 45(3):60-67.

Zhao, Shanyang, Sherri Grasmuck, and Jason Martin. 2008. "Identity Construction on Facebook: Digital Empowerment in Anchored Relationships." Computers in Human Behavior 24(5):1816-36.

Zhao, Xuan, Niloufar Salehi, Sasha Naranjit, Sara Alwaalan, Stephen Voida, and Dan Cosley. 2013. "The Many Faces of Facebook: Experiencing Social Media as Performance, Exhibition, and Personal Archive." Pp. 1-10 in Proceedings of the SIGCHI conference on human factors in computing systems.

Zhu, Wei, Daqing Zheng, Wencan Wang, and Hanhui Zhou. 2014. "The Differences between Weibo and Wechat: The Evidence from the Social Capital Theory." Journal of Intelligence 6:138-43.

ABOUT THE CONTRIBUTOR(S) 
Xiaoli Tian is an Associate Professor in the Department of Sociology at the University of Hong Kong. She obtained her Ph.D. in Sociology from the University of Chicago. Her research interests include how preexisting knowledge paradigms and cultural norms influence the way people respond to unexpected transformations of their everyday routines. This interest is reflected in her two main lines of research: medical knowledge as situated practices and social interaction. Her writings have been published in American Journal of Sociology, Sociological Forum, Qualitative Sociology, Modern China, Information, Communication and Society, Journal of Contemporary Ethnography, Media, Culture and Society, Symbolic Interaction, and Journal of Social and Personal Relationships.

Yanan Guo is a PhD Candidate at the Department of Sociology, The University of Hong Kong. She obtained her bachelor's degree in sociology, translation, and journalism and media studies at The University of Hong Kong and had worked as a media analyst in the tech industry. Her research interests include science and technology studies, social interaction, social innovation, and digital entrepreneurship.

\section{APPENDIX}

Figure 1. Schematic of Online Acquaintance Community 


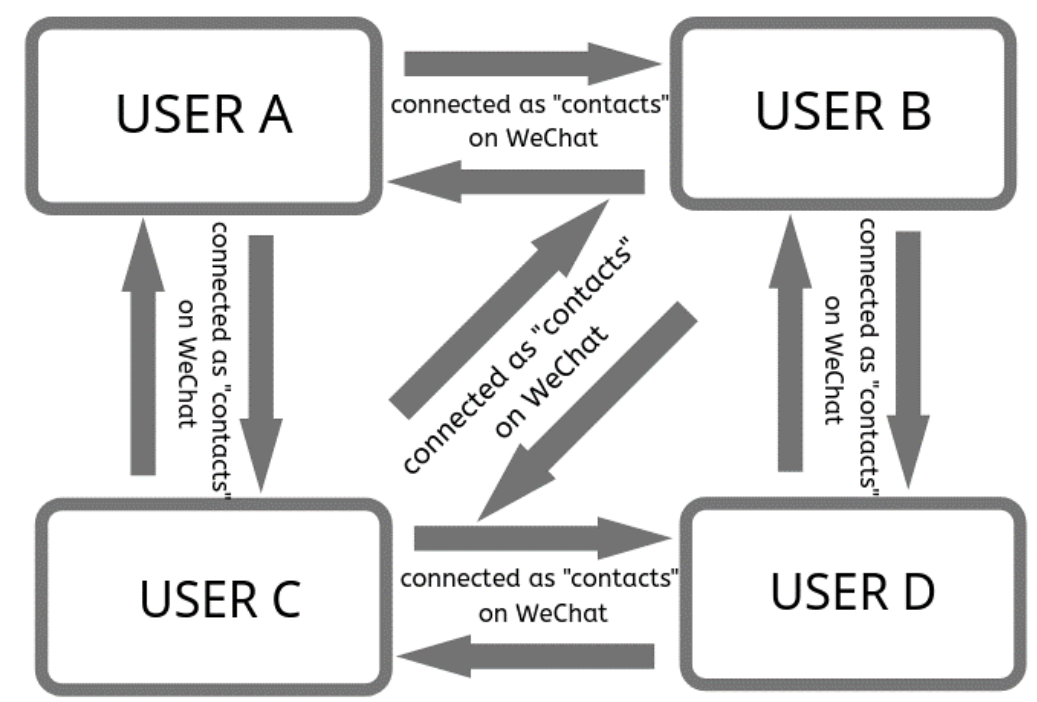

- If User B posts a "Moment", User A, C, D can all leave comments under the post. However, only User C's comments can be displayed to both User A, B and D. User A's comments can only be displayed to User B and C, and User D's comments can only be displayed to User B and C. User A and User D cannot see each other's comments under User B's posts since they are not connected as contacts on WeChat. 